\title{
Evaluación final: ¿sirve el examen MIR?
}

\author{
Francisco J. Aspa Marco ${ }^{a}$, Felipe Rodríguez de Castro ${ }^{b}$
}

\section{Introducción}

La realización de una prueba única, de carácter nacional y de diseño centralizado tras la finalización de los estudios de grado, con objeto de seleccionar $\mathrm{u}$ ordenar a los aspirantes a acceder a un programa de formación especializada, refleja el actual paradigma en el que se evalúa y recompensa fundamentalmente los conocimientos adquiridos durante los estudios universitarios. No obstante, el planteamiento de la pregunta que encabeza este escrito no es inocente y deja traslucir algunas inconsistencias que se tratarán de hacer patentes a lo largo del artículo. Además, se propondrá exponer las claves que permitan plantearnos otro paradigma, basado en la capacidad de aprender.

En la figura 1 se señalan los principales pasos que el estudiante de medicina debe dar para convertir su vocación en profesión y para seguir manteniendo sus conocimientos actualizados a lo largo de su vida. En este sencillo esquema se describe que, tras una prueba de acceso a la Facultad de Medicina (examen de selectividad), el estudiante cursará sus estudios de grado para, al terminar éstos, ya como médico, realizar el examen de médico interno residente (MIR), y así optar a una plaza que le permita obtener un título de especialista y lo capacite legalmente para ejercer en el sistema sanitario público. La competencia alcanzada por el especialista representará el grado en el que ese médico puede utilizar los conocimientos, las aptitudes, las actitudes y el buen juicio asociados a la profesión, para poder desempeñarse de manera eficaz en todas las situaciones que corresponden al campo de su práctica pro- fesional. El profesional tiende a la excelencia y el profesionalismo exige acceder permanentemente a los valores de la profesión y a mantener el máximo nivel personal de competencia exigible en cada momento. Todas las acciones encaminadas a ello configuran el desarrollo profesional continuo, que se podría definir como el proceso mediante el cual los profesionales adquieren, mantienen y mejoran sus conocimientos, sus habilidades y sus actitudes, lo que les permite continuar su ejercicio profesional de forma competente, esto es, con el nivel de calidad exigible en cada momento. Se trataría, en definitiva, de mantener el clásico esquema aristotélico: aprender a pensar, aprender a decidir, aprender a ser.

\section{Análisis del sistema actual}

Situados en este esquema, la primera pregunta que deberíamos plantearnos es la clásica ante cualquier examen: ¿qué se valora en el examen MIR? Antes de responder sería adecuado realizar una breve reflexión. Se acepta que las instituciones que detentan la facultad de otorgar títulos han de ser responsables de aquello que 'certifican', pero si observamos el esquema anterior, parecería que no nos fiásemos de la capacidad de la universidad para acreditar y quisiéramos asegurar la validez de los títulos que otorga, la adecuación de la formación recibida. No olvidemos que si un médico no 'aprueba' el examen, es decir, si no obtiene una puntuación suficiente que le permita acceder a la formación especializada, su reconocimiento como médico titulado sigue siendo válido.

\footnotetext{
Director Médico, Hospital Universitario de la Princesa. Profesor asociado, UAM.

b Decano de la Facultad de Ciencias de la Salud. Universidad de Las Palmas de Gran Canaria.

E-mail jaspa@separ.es
} 


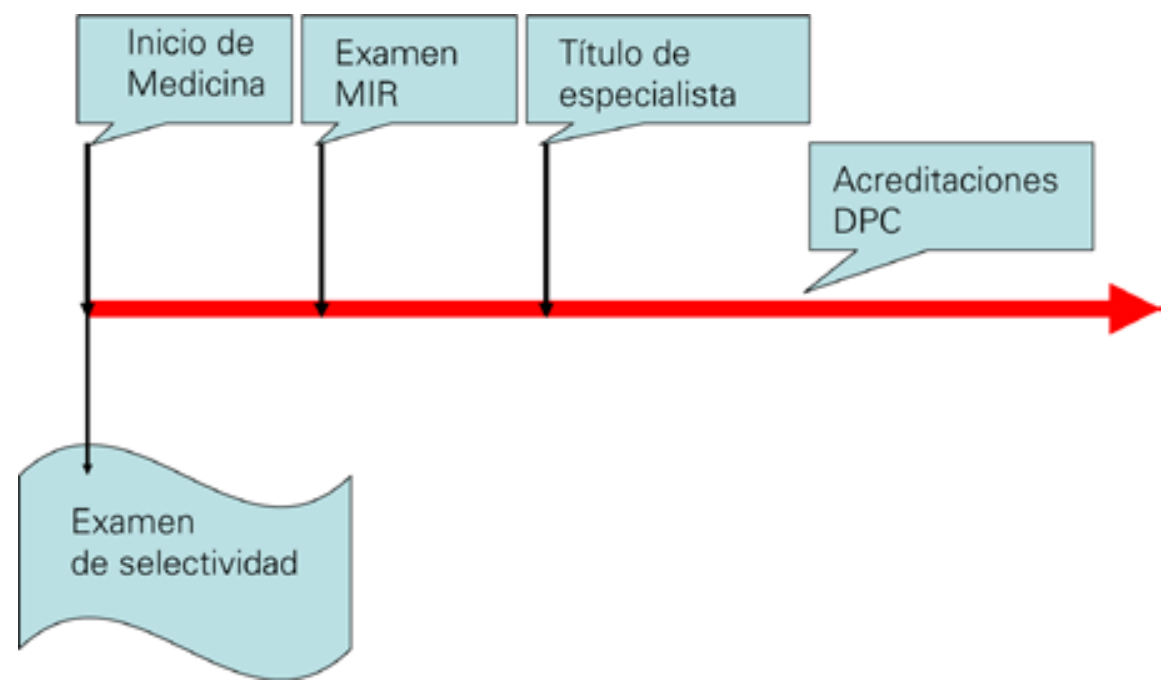

Figura 1. Pasos a dar en la titulación/formación como médico.

Se podría argumentar que una prueba como el examen MIR no quiere evaluar los conocimientos adquiridos, sino otro tipo de conocimientos y habilidades que no están suficientemente normalizados o que pertenecen a lo que podemos denominar 'currículum oculto'. Es decir, que lo que se pretende valorar realmente son otras capacidades, necesarias para el ejercicio de la profesión médica, como habilidades de comunicación o de relación, empatía, inteligencia emocional, etc. Sin embargo, resulta evidente que un instrumento evaluador como el que se emplea en el examen MIR no permite una evaluación adecuada de estos aspectos ni es este su objetivo declarado.

A pesar de todo, además de servir de llave para acceder a una formación especializada, el examen MIR tiene otros valores añadidos que conllevan claramente una predicción de gran disfunción entre las necesidades asistenciales de la población y la necesidad de especialistas que genera nuestro modelo sanitario [1]. Como ejemplos se podrían mencionar el negocio de las academias preparatorias, su papel como numerus clausus real para regular el mercado de especialidades, la formación de médicos de otras nacionalidades, el actuar de facto como un sistema de validación de títulos, etc.

Conviene que volvamos al esquema de la figura 1 y reflexionemos un poco más: ¿qué medicina se puede ejercer sin tener una especialidad vía MIR? Desde luego, ninguna ligada a la clínica si aten- demos a las directrices europeas para ejercer en la sanidad pública [2]. En España se condicionó el ejercicio de las actividades de médico general, en el marco de su régimen nacional de seguridad social, a la posesión del título de especialista en Medicina Familiar y Comunitaria.

Las siguientes preguntas que surgen son: ¿qué relación tengo entre la oferta y la demanda?, ¿cuántas plazas MIR oferto y cuántas solicitudes tengo? En la figura 2 se muestran datos muy esclarecedores. El inicio de la formación especializada vía MIR, junto con un excesivo número de licenciados, supuso para nuestro país, a finales de los años setenta, un cambio en la estructura de la profesión hasta entonces desconocido. Sólo unos pocos accedían a la formación especializada. El resto, asimilado a un 'cierto' segundo nivel, buscó un hueco laboral básicamente en la atención primaria, en las zonas rurales y en las bolsas del paro. En numerosas ocasiones actuaron como médicos especialistas, aunque sin título, lo que más tarde originó otra situación paradójica a la que hubo que poner remedio: los médicos especialistas sin título oficial ('MESTOS').

En conjunto, la cifra de candidatos médicos presentados al examen ha pasado de 23.890 en 1983 a 6.726 en el año 2011. También se ha modificado su procedencia, de forma que, por ejemplo, para el caso de las plazas adjudicadas a Medicina Familiar y Comunitaria, de un total de 1.648 


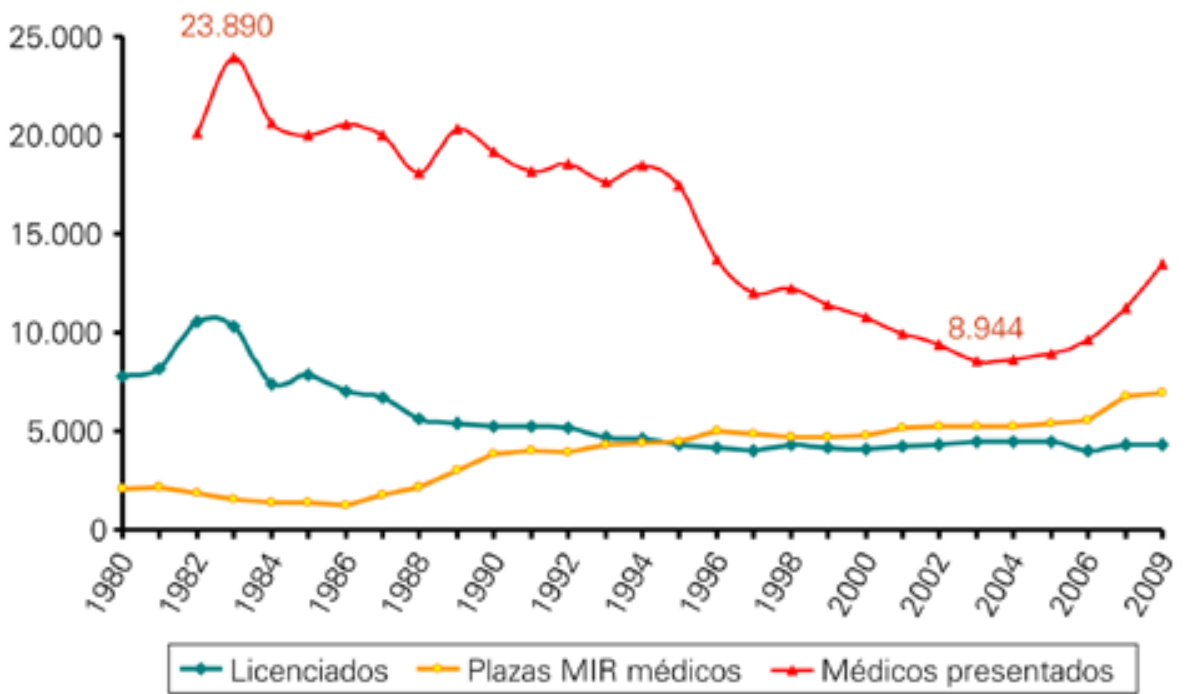

Figura 2. Sistema MIR: licenciados en Medicina, plazas MIR y número de presentados.

plazas en 2005-2006, el 1,6\% correspondía a médicos comunitarios no españoles y el $11,8 \%$ a no comunitarios. Para el año 2009-2010, de un total de 1.904 plazas, el $4,2 \%$ correspondía a comunitarios y el $40,5 \%$ (771 plazas) a no comunitarios.

El examen MIR es, posiblemente, un punto estratégico en la vida profesional de los médicos y tiene una de las mayores potencialidades de promover cambios en cascada en su entorno, con repercusiones tanto en la etapa de grado como en la de especialización [1]. Los autores de este artículo, por su dedicación profesional, llevan más de 20 años asistiendo a la llegada a sus centros de diferentes promociones de residentes e, independientemente de la especialidad, de la procedencia o de las diversas circunstancias que concurren, una afirmación es constante: el tipo de examen MIR actualmente existente actúa de forma determinante sobre el tipo de enseñanza que se realiza en las universidades. No sólo modifica el sistema de enseñanza, sino que los alumnos del grado o licenciatura filtran aquellos aspectos de su formación según que éstos se acomoden al tipo de examen al que se van a someter, primando los aspectos que consideran útiles y soslayando los que consideran ajenos a este fin último: aprobar el examen y obtener una plaza de formación especializada. Si la universidad vive de espaldas a esta realidad constatable, puede darse el caso de que elabore estrategias pedagógicas innovadoras, pero que éstas se desarrollen en una especie de limbo, con unos estudiantes que pueden llegar a considerar su formación universitaria como un trámite completamente alejado de la realidad. De hecho, se está asistiendo a fenómenos preocupantes, como la utilización de los manuales elaborados por las academias de preparación para la prueba MIR como libros de uso habitual por parte de estudiantes de facultades prestigiosas de nuestro país, o la iniciación de la preparación al examen en estas academias durante el último año de la licenciatura, convirtiendo esta actividad en prioritaria en relación con sus obligaciones académicas como pregraduados.

Los análisis más habituales acerca de la prueba de acceso MIR se centran en examinar aspectos menos trascendentes, como el número de preguntas por especialidad, destacando que la distribución de éstas responde predominantemente a una estrategia cuantitativa de reparto de materias y no a su pertinencia o a un análisis de competencias médicas predeterminadas. Otro enfoque común es analizar la oferta de plazas por especialidad o establecer un ranking de universidades en base a criterios tan arbitrarios como el número de estudiantes que se sitúan entre los 100 primeros puestos, lo que sin duda se presta a manipulaciones interesadas, ya que no se tiene en cuenta la razón entre los primeros puestos y el número de egre- 
Tabla. Siglo XXI: nuevo paradigma en la gestión del conocimiento.

Desarrollar estrategias de conocimiento

Reglas operativas claras que nos permitan:

Gestionar el conocimiento

Gestionar nuestra capacidad de aprender

sados. En todo caso, el Ministerio de Educación elabora su propio ranking en el que los expertos consideran unos percentiles distintos y, aparentemente, más reflexionados.

Vázquez et al [1] realizan un análisis DAFO sobre el examen MIR que mejora nuestra comprensión de estos aspectos. A modo de resumen, los autores extraen las siguientes conclusiones:

- Debilidades: 1 . Sólo analiza algunos aspectos cognitivos; 2 . La validez no es óptima a causa del número de preguntas que contiene; 3 . Los hospitales son receptores pasivos; 4 . Los exámenes tienen un formato escrito clásico.

- Amenazas: 1. El estudiante centra sus estudios en el examen MIR; 2. La preparación del MIR se hace con proveedores externos.

- Fortalezas: 1. Afecta a todo el colectivo médico; 2. Transparencia, equidad y accesibilidad.

- Oportunidades: 1. Nueva propuesta de licenciatura médica; 2 . Nuevas metodologías de formación y entrenamiento; 3 . Tecnologías de información y comunicación.

\section{Otros planteamientos}

En otras latitudes se plantean otro tipo de prueba de acceso a la formación especializada. El ejemplo más conocido es el desarrollado en Estados Unidos, denominado United State Medical Licensing Examination (USMLE) [3], que lo constituyen tres fases distintas: la primera se centra en las ciencias básicas aplicadas a problemas clínicos; la segunda fase evalúa el conjunto de conocimien- tos y habilidades necesarios para la práctica clínica, y la tercera aborda aspectos relacionados con esta última y que un médico general debe poder afrontar en su etapa inicial. En conjunto, la prueba tiene alrededor de 1.200 preguntas, 11 estaciones con enfermos simulados y 20 casos clínicos completos desarrollados por ordenador. Se requieren cinco días, con jornadas de ocho horas, para su ejecución, y al contrario que el examen MIR, no existe una prueba única y simultánea, sino que los candidatos disponen de un año para pasar las etapas mencionadas.

En nuestro país, en la convocatoria anterior, se introdujeron una serie de modificaciones con respecto a la estructura tradicional del examen MIR, que básicamente consistieron en la inclusión de supuestos clínicos con apoyo iconográfico. Aunque sólo ha sido un tímido avance, supone un paso en la dirección correcta en la que podría profundizarse aprovechando el desarrollo tecnológico a nuestro alcance. Además, y con objeto de dar cumplimiento a lo contemplado en la Ley de Ordenación de las Profesiones Sanitarias (LOPS), una alternativa no desdeñable es que, en un próximo futuro, las facultades realizaran una prueba al final del grado, de diseño central y ejecución descentralizada, que con las debidas garantías de equidad evaluara aspectos relacionados con las habilidades clínicas y de comunicación, y que tuviera validez académica reconocida para el acceso a la formación especializada.

\section{Nuevo paradigma: acrecentar nuestra capacidad de aprender}

En pocas profesiones, la renovación continua del conocimiento se hace tan necesaria como en la medicina. Sin que esté próxima aún la edad de jubilación de los autores, cuando ambos comenzamos a ejercer la medicina no existía por ejemplo la tomografía axial computarizada (TAC) ni, por supuesto, la tomografía por emisión de positrones-TAC; tampoco se conocían la mayor parte de los antibióticos que ahora manejamos de forma más habitual, ni la familia de los antiinflamatorios ligados a la inhibición del factor de necrosis tumoral o los nuevos fármacos oncológicos, por citar sólo algunos ejemplos. Lo mismo podría comentarse a propósito de las modificaciones en los 
paradigmas fisiopatológicos, genéticos o bioquímicos a los que continuamente asistimos.

Resulta absolutamente imprescindible que los médicos incorporen en su método de trabajo estructuras estables que les permitan mantener operativa su capacidad de aprender (Tabla). Es básico que en el método de estudio, ya en la etapa de formación universitaria, se desarrollen estrategias que permitan gestionar el conocimiento y nuestra capacidad de incrementarlo, modularlo y matizarlo.

\section{Bibliografía}

1. Vázquez G, Murillo-Cabezas F, Gómez J, Martín C, Chaves J, Peinado JL. El examen MIR, su cambio como una opción estratégica. Educ Med 2008; 203-6.

2. Directiva $2005 / 36 /$ CE del Parlamento Europeo y del Consejo de 7 de Septiembre de 2005, relativa al reconocimiento de cualificaciones profesionales.

3. United State Medical Licensing Examination. URL: http:// www.usmle.org. [17.10.2010]. 